\title{
Circulating CD147 Predicts Mortality in Advanced Hepatocellular Carcinoma
}

Aimei Lee ${ }^{1,2 \dagger}$,Anthony Rode ${ }^{3 \dagger}$, Amanda Nicoll ${ }^{4 \dagger}$, Annette E Maczurek ${ }^{1,2}$, Lucy Lim ${ }^{5}$, Seok Lim ${ }^{3}$,

Peter Angus ${ }^{5}$, Ian Kronborg ${ }^{6}$, Niranjan Arachchi ${ }^{6}$, Alexandra Gorelik ${ }^{7}$, Danny Liew ${ }^{7}$, Fiona J Warner $^{1,2}$, Geoffrey W McCaughan ${ }^{1,2,8}$, Susan V McLennan ${ }^{2,9}$, Nicholas A Shackel ${ }^{1,2,8}$.

${ }^{\dagger}$ Equal first authors.

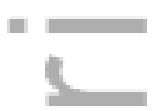

${ }^{1}$ Centenary Institute of Cancer Medicine and Cell Biology, Camperdown, NSW Australia

${ }^{2}$ Sydney Medical School, The University of Sydney, Camperdown, NSW, Australia

${ }^{3}$ Department of Gastroenterology and Hepatology, Royal Melbourne Hospital, Melbourne, VIC,

Australia

${ }^{4}$ Department of Gastroenterology and Hepatology, Box Hill Hospital, Box Hill, VIC, Australia

5 Victorian Liver Transplant Unit and Department of Gastroenterology, Austin Hospital, Melbourne, VIC, Australia

${ }^{6}$ Department of Gastroenterology, Western Hospital, Footscray, VIC, Australia.

${ }^{7}$ Melbourne EpiCentre, University of Melbourne and Melbourne Health, Parkville, VIC, Australia ${ }^{8}$ A.W. Morrow Gastroenterology and Liver Centre, Royal Prince Alfred Hospital, Camperdown, NSW, Australia

${ }^{9}$ Department of Endocrinology, Royal Prince Alfred Hospital, Camperdown, NSW, Australia

CORRESPONDING AUTHOR: Nicholas A. Shackel

Address: Centenary Institute of Cancer Medicine and Cell Biology, Johns Hopkins Drive,

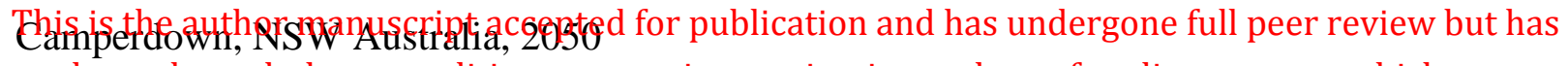
not been through the copyediting, typesetting, pagination and proofreading process, which may

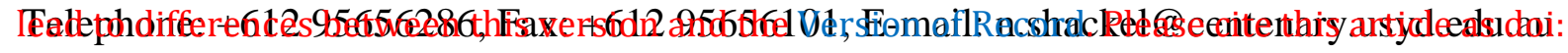
10.1111/JGH.13148

This article is protected by copyright. All rights reserved. 
Short Running Title: CD147 predicts mortality in liver cancer

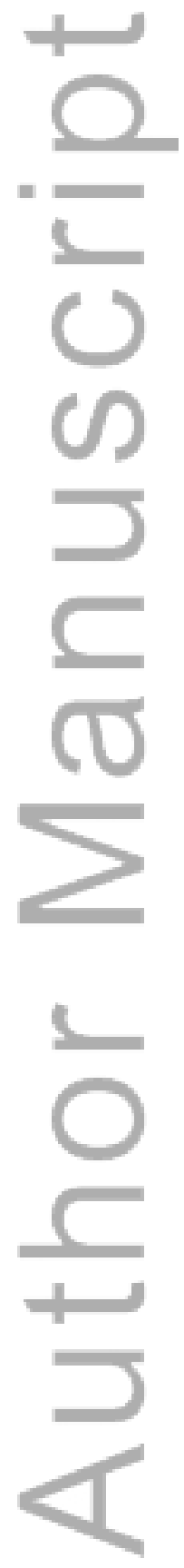




\section{ABSTRACT}

Background \& Aims: The glycoprotein CD147 has a role in tumor progression, is readily detectable in the circulation and is abundantly expressed in HCC. Advanced HCC patients are a heterogeneous group with some individuals having dismal survival. The aim of this study was to examine circulating soluble CD147 levels as a prognostic marker in HCC patients.

Methods: CD147 was measured in 277 patients (110 HCC, 115 chronic liver disease and 52 nonliver disease). Clinical data included etiology, tumor progression, Barcelona Clinic Liver Cancer (BCLC) stage and treatment response. Patients with HCC were stratified into two groups based upon the $75^{\text {th }}$ percentile of CD147 levels (24ng/mL).

Results: CD147 in HCC correlated inversely with poor survival $(P=0.031)$. Increased CD147 predicted poor survival in BCLC stages C and D $(P=0.045)$ and CD147 levels $>24 \mathrm{ng} / \mathrm{mL}$ predicted a significantly diminished 90-day and 180-day survival time (hazard ratio $(\mathrm{HR})=6.1$; 95\% confidence interval (CI):2.1-63.2; $P=0.0045$ and HR=2.8; 95\% CI:1.2-12.6; $P=0.028$, respectively). In BCLC stage C, CD147 predicted prognosis; levels > 24ng/mL were associated with a median survival of 1.5 months compared to 6.5 months with CD147 levels $\leq 24 \mathrm{ng} / \mathrm{mL}$ $(P=0.03)$. CD147 also identified patients with a poor prognosis independent from treatment frequency, modality and tumor size.

Conclusions: Circulating CD147 is an independent marker of survival in advanced HCC. CD147 requires further evaluation as a potential new prognostic measure in HCC to identify patients with advanced disease who have a poor prognosis.

KEYWORDS: CD147, hepatocellular carcinoma, prognosis, biomarker 


\section{INTRODUCTION}

Hepatocellular carcinoma (HCC) is the fifth most common cancer diagnosis worldwide ${ }^{1}$, characterized by rapid progression, poor prognosis and frequent recurrence. There is a strong and consistent association between HCC and cirrhosis ${ }^{1-3}$. In HCC complicating cirrhosis there are diminished options for treatment resulting in the need for accurate predictive markers to improve survival ${ }^{2}$.

CD147 is a cell surface transmembrane glycoprotein involved in cellular adhesion, cell-cell and cell-extracellular matrix (ECM) interactions ${ }^{4,5}$. CD147 has been widely studied in tumor-matrix interactions, regulating the expression of ECM degrading enzymes called matrix metalloproteinases (MMPs) ${ }^{6,7}$ and promotes tumor angiogenesis and proliferation ${ }^{8}$. Importantly, increased expression of cancer-associated CD147 predicts aggressive behavior and poor outcomes $^{9-11}$. CD147 is also expressed as a soluble form ${ }^{12}$, readily detectable in serum and plasma, and released via shedding of microvesicles or proteolytic cleavage ${ }^{13,14}$.

Advanced stages of HCC are associated with higher intrahepatic CD147 mRNA levels ${ }^{9}$ and intrahepatic CD147 is an independent predictor of poor survival in HCC patients who had undergone tumor resection ${ }^{15}$. Post-liver transplantation, CD147 predicted tumor recurrence and poor prognosis when combined with CD34 expression ${ }^{16}$. Licartin $\left(\left[{ }^{131} \mathrm{I}\right]\right.$ metuximab injection), a therapeutic anti-HCC antibody targeting CD147 ${ }^{17}$, significantly inhibits tumor cell growth in-vitro and in-vivo in animals ${ }^{15}$.

Given that CD147 plays a role in HCC progression, we hypothesized that it could provide 
prognostic information. The aim of this study was to establish the usefulness of soluble CD147 (sCD147) in predicting HCC prognosis. We prospectively examined sCD147 plasma levels in HCC patients and compared the results to a non-HCC chronic liver disease (CLD) cohort and a control cohort without liver disease. We also evaluated the relationship between sCD147 and clinical markers of HCC extent and progression.

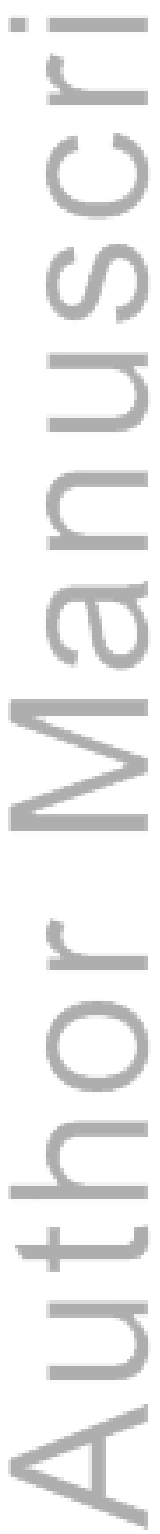




\section{METHODS}

\section{Study population and Ethics}

This prospective study enrolled patients from the Royal Melbourne, Austin and Western hospitals in Melbourne, Australia, between June 2008 and December 2010. The HCC cohort had the confirmed diagnosis of HCC through characteristic imaging or histology. Patients were included in the CLD cohort based on a combination of clinical, biochemical, radiological and liver histological findings. Control patients with no history of liver disease were recruited from the surgical preadmission clinic. Subjects were excluded from this control cohort if they had: evidence of a current infection or neoplastic process; significant medical co-morbidities; suspicion of liver disease on history, examination, biochemistry or imaging; or long term daily alcohol intake greater than $30 \mathrm{~g}$ per day.

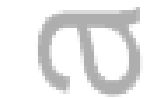

All participants provided written, informed consent. The study protocol conforms to the ethical guidelines of the 1975 Declaration of Helsinki and was approved by the relevant institutional ethics review committees at each site.

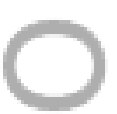

\section{Clinical Assessment}

Clinical history, imaging and examination were undertaken at the time of enrolment. Data collected at enrolment and follow-up (up to one year post-enrolment) included: complications due to cirrhosis, hospital admissions, Child-Turcotte-Pugh (CTP) score and Model for End Stage Liver Disease (MELD) score. Date of final follow-up, death, transplantation or last review was also recorded. 
$\underline{\text { Samples were taken around the time of presentation to a multidisciplinary meeting when there was }}$ consideration of treatment options. All patients with HCC were offered treatment with locoregional therapy (LRT) or sorafenib. HCC activity was determined by the presence of arterial enhancement on arterial phase computed tomography (CT) scanning with portal phase washout using modified Response Evaluation Criteria in Solid Tumors (mRECIST) ${ }^{18,}{ }^{19}$. Imaging was performed at 3 monthly intervals or 1 month after LRT to assess efficacy. Based on the mRECIST criteria two HCC sub-categories were defined: a) HCC without ongoing arterial enhancement and complete response to treatment (referred to as 'HCC with a complete response to treatment (HCC with CR) subgroup') or b) HCC with current arterial enhancement and/or disease progression (referred to as 'HCC without a complete response to treatment (HCC without CR) subgroup'). Patients without LRT were assessed by mRECIST for categorization as with CR or without CR. Patients with HCC who died due to liver causes, developed extrahepatic spread or multifocal lesions were categorized into the HCC without CR subgroup. HCC progression was assessed using Barcelona Clinic Liver Cancer (BCLC) staging, biochemical tests and imaging.

\section{Biochemical analyses}

Blood samples were obtained at study enrolment and analyzed for liver function and $\alpha$-fetoprotein. Additional samples were collected in EDTA vacutainers, centrifuged at $1500 \mathrm{~g}$ for $10 \mathrm{~min}$ and plasma stored at $-80^{\circ} \mathrm{C}$ prior to analysis for sCD147 at the Centenary Institute, Sydney, Australia.

sCD147 levels were measured in plasma using an enzyme-linked immunosorbent assay (ELISA) as previously described ${ }^{20}$. ELISAs were performed blinded to the clinical data. Recombinant human CD147 standards (R\&D Systems, Minneapolis, USA) and plasma samples were analyzed 
in triplicate. Mean concentrations of the samples were calculated and samples with a between triplicate coefficient of variation greater than $15 \%$ were re-analyzed.

\section{Immunohistochemistry}

Paraffin-embedded human liver (cirrhotic alcoholic liver disease (ALD) $n=4$, cirrhotic ALD with HCC $n=4$, and non-diseased $n=4$ ) was examined for the expression of CD147 as previously described $^{21}$.

\section{Statistical analyses}

Data was analyzed using Stata 10 (StataCorp, Texas, USA). Comparisons between cohorts were performed using Chi-squared tests for categorical data and ANOVA with Kruskal-Wallis test or ttest with Mann-Whitney test for continuous, non-normally distributed data. Spearman’s rank correlation tests were used to correlate data. Multivariate Cox regression analysis was used to determine factors associated with patients' death. $P$-values $<0.05$ were considered statistically significant for all tests. Multivariate analysis was undertaken for HCC disease activity (CR or nonCR), age (50 years), gender, underlying cause of disease (ALD, HCV, HBV, NAFLD and other) and ethnicity (Anglo-Saxon, Asian, Mediterranean and Other). 


\section{RESULTS}

\section{Study population}

Patient stratification and cohort composition is shown in Figure 1. HCC was diagnosed at a median of 13.1 months before study enrolment (interquartile range (IQR):4.2-28.8). The HCC with

CR subgroup (37.3\% of the HCC cohort) median time since the last HCC treatment was 11.2 months (IQR:5.1-31.8). Overall the CLD and HCC cohorts were similar. However, there were more individuals with a CTP C score in the CLD cohort $(29.6 \%$ vs. $10.9 \%, P<0.05)$ and mortality was greater in the HCC cohort (40 vs. 17 deaths, $P<0.01$ ). Further, 91.8\% of patients with HCC in this study had cirrhosis.

Baseline patient demographics and cause of liver disease are shown in Table 1. The main underlying cause of liver disease was hepatitis C virus (HCV) in both cohorts. Median CTP and MELD scores were higher in the CLD cohort than HCC cohort. The CLD cohort was younger than the control cohort (59.3 vs 67.1 years, $P<0.001)$. There was no difference in age comparing the CLD and HCC cohorts. There were no significant differences between all cohorts in gender, ethnicity or diabetes.

\section{Mortality and sCD147 levels}

In the HCC cohort, death was related to liver disease and/or HCC in 97.5\% of patients; median survival time from study enrolment of 7.1 months (IQR:5.2-9.1). Expectedly, the majority of patients who died (92.5\%) were within the HCC without CR subgroup. Survival in this subgroup was a median of 6.4 months (IQR:3.5-8.7). 
sCD147 levels were highest in the control cohort (25.6ng/mL, IQR:11.1-34.9), and equivalent in the CLD (17.7ng/mL, IQR:12.9-23.9) and HCC (16.0ng/mL, IQR:12.2-23.4) cohorts $(P=0.1)$. As CD147 expression can predict tumor behavior and outcomes ${ }^{9,15}$, we determined the relationship of sCD147 to CLD and HCC outcomes. sCD147 correlates with death in HCC but not CLD (Figure 2A). Further, within the HCC cohort, sCD147 predicted death in advanced disease (BCLC stage C and D) but not early disease (BCLC stage A and B), shown in Figure 2B.

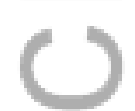

There was a significant survival advantage at early time points with lower sCD147 levels in the HCC cohort when patients were categorized into two groups based upon the $75^{\text {th }}$ percentile of sCD147 levels; patients with sCD147 levels $>24 \mathrm{ng} / \mathrm{mL} \underline{(\mathrm{n}=25)}$ and levels $\leq 24 \mathrm{ng} / \mathrm{mL} \underline{(\mathrm{n}=85)}$ (Figures 3 and 4). sCD147 levels $>24 \mathrm{ng} / \mathrm{mL}$ did not predict a survival disadvantage at the 1-year follow-up time-point (hazard ratio $(\mathrm{HR})=1.2 ; 95 \%$ confidence interval (CI):0.6-2.6; $P=0.7$ ). However, at the earlier time-points of 90-days and 180-days, sCD147 levels $>24 \mathrm{ng} / \mathrm{mL}$ predicted a significantly diminished survival time $(\mathrm{HR}=6.1 ; 95 \% \mathrm{CI}: 2.1-63.2 ; P=0.0045$ and $\mathrm{HR}=2.8 ; 95 \%$

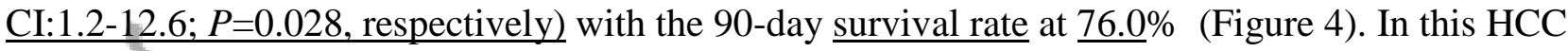
subgroup, the median survival time from study enrolment was 2.6 months (IQR:0.9-5.9). sCD147 levels $\leq 24 \mathrm{ng} / \mathrm{mL}$ was associated with a significantly higher survival at 90 -days $(\underline{96.5} \%, P<0.01)$ and 180-days (88.2\%, $P<0.05)$. In the HCC without CR subgroup, sCD147 predicted mortality at 90-days with a sensitivity of $78 \%$, specificity of $88 \%$, negative predictive value (NPV) of 96\%, positive predictive value (PPV) of $50 \%$ and likelihood ratio (LR) of 6.5. The area under the

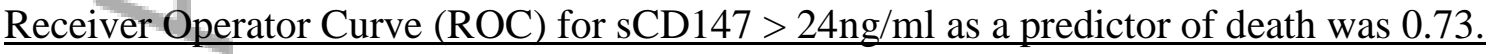

$\underline{\text { AFP does not correlate with sCD147 irrespective of liver disease stage or stratification of sCD147 }}$ 
(see Table S1). Further, with HCC a rising AFP does not correlate with sCD147 ( $\mathrm{r}=0.1781$ $\underline{P=0.07) \text { (Table S1). }}$

\section{Multivariate analysis, HCC disease progression, portal hypertension and sCD147 levels}

Multivariate regression analysis of the HCC cohort adjusted for HCC disease activity, age, gender, underlying cause of disease and ethnicity demonstrated no correlation between sCD147 or tumor scores $(P=0.296)$, indicating that sCD147 is predicting poor HCC outcomes independent of deteriorating liver function or tumor characteristics. Further, there was no correlation between time to death and sCD147 levels $>24 \mathrm{ng} / \mathrm{mL}$ within the CLD cohort $(P=0.269)$. Death was related to worsening liver function, with the median MELD score increasing from 16.5 (IQR:13.1-23.6) at enrolment to 23.8 (IQR:16.6-29.0, $P=0.05)$.

\section{(1)}

Levels of $\mathrm{sCD} 147>24 \mathrm{ng} / \mathrm{mL}$ in the HCC without CR subgroup predicted disease progression at 90-days with a specificity of $95 \%$, NPV of $75 \%$, PPV of $86 \%$ and LR of 9.2. Examination of tumor characteristics demonstrated that increasing tumor size correlated with higher sCD147 levels ( $\mathrm{r}=0.228, P=0.017)$. Further, patients with tumor vascular invasion also had higher sCD147 levels $(P=0.009)$ and poor outcomes. Importantly, no other tumor characteristics including tumor number, $\alpha$-fetoprotein levels, presence of metastasis and histological staging showed any significant correlation with sCD147.

In BCLC stages A and B, sCD147 did not predict mortality. In BCLC stage C, patients with sCD147 levels $>24 \mathrm{ng} / \mathrm{mL}$ had a median survival of 2.6 months (IQR:0.9-5.1), in contrast to a survival of 6.5 months (IQR:5.9-8.6) in patients with sCD147 levels $\leq 24 \mathrm{ng} / \mathrm{mL}(P=0.03)$ (Figure 
3). Importantly, the ability of sCD147 to predict mortality in BCLC stage C disease was independent of tumor size, number and treatment modality. In BCLC stage D $(n=13)$, the median survival of patients with sCD147 levels $>24 \mathrm{ng} / \mathrm{mL}$ was not different from patients with sCD147 levels $\leq 24 \mathrm{ng} / \mathrm{mL}$ (1.5 months vs 4.5 months; $P=0.547)$.

.

We also specifically examined if the presence of portal hypertension (assessed by ascites and/or imaging) predicted CR. Irrespective of BCLC staging there was no difference in treatment CR stratified for the presence or absence of portal hypertension. In BCLC stages A to C there was no significant difference in portal hypertension, and in BCLC stage D, all individuals without CR had portal hypertension.

\section{HCC treatment, liver transplantation and sCD147 levels}

The different treatments for HCC are listed in Table 1. LRTs for HCC included: transcatheter arterial chemoembolization (TACE) ( $n=68$, mean of 2.0 episodes), radiofrequency ablation ( $n=40$, mean of 1.5 episodes), percutaneous ethanol injection ( $n=11$, not repeated) and surgical resection ( $n=20$, not repeated). Response to treatment was defined by mRECIST criteria. Patients without CR had significantly lower sCD147 levels compared to those that did not receive LRT (16.19ng/mL (IQR:12.29-20.87) vs 27.02ng/mL (IQR:17.94-31.99), $P=0.020)$. Six patients had sorafenib as initial therapy, with 14 patients having sorafenib after disease progression. There was no change in patient outcome with high sCD147 levels according to the treatment modality received.

Four HCC patients with CR met the Milan criteria ${ }^{22}$ and underwent liver transplantation. Only one 
patient had a sCD147 level > 24ng/mL $(56.7 \mathrm{ng} / \mathrm{mL})$ at enrolment, and received a liver transplant three months post-enrolment. The explanted liver demonstrated six lesions and lymphovascular invasion, which was not detected on triple phase CT imaging two months prior to transplantation. Within five months post-transplantation, multifocal liver tumors recurred and death occurred at 10 months post-transplant. No other transplanted patient (all with sCD147 levels $\leq 24 \mathrm{ng} / \mathrm{mL}$ ) had $=$ post-transplant HCC recurrence in the follow-up period.

\section{Immunohistochemistry of CD147}

Representative panels of immunostained liver sections to assess cellular CD147 expression are shown in Figure 5. No differences were observed in the pattern of CD147 expression on intrahepatic cell populations in the non-diseased, cirrhotic or cirrhotic with HCC tissue examined. The staining shows abundant CD147 expression on hepatocytes, leukocytes and biliary epithelium. There is negligible expression in the fibrous septa where activated hepatic stellate cells are located. Comparing equivalent antibody concentrations, staining protocols and imaging settings, there was consistently more intense CD147 expression seen in all HCC samples compared to cirrhosis without HCC and non-diseased tissue.

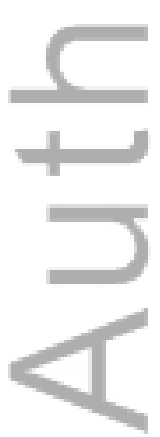




\section{DISCUSSION}

The main finding of this study was that an increased plasma sCD147 level identified patients with more advanced HCC and predicted mortality. This appears to be due to tumor biology as sCD147 levels were independent of the number of lesions, treatment modality and portal hypertension. There was also a correlation of sCD147 with increasing tumor size. sCD147 may assist in the identification and management of HCC patients with a poor prognosis, particularly individuals with evidence of tumor activity following LRT. Identifying such patients will lead to more effective palliation and earlier adoption of differing treatment regimes.

Identifying patients without a radiological response to treatment is necessary to direct further treatment. Further, the utility of any biomarker is enhanced if integrated with established clinical management. This has been demonstrated in our study using a sCD147 level of 24ng/mL to stratify risk. Selection of this sCD147 level was based on the BCLC stage C group being 25.5\% of the total HCC cohort and $24 \mathrm{ng} / \mathrm{mL}$ being the $75^{\text {th }}$ percentile of all sCD147 levels. All HCC deaths within 90-days of study enrolment occurred in the HCC without CR subgroup. High sCD147 levels were specific for early death and predicted HCC disease progression within 90-days. In the HCC with CR subgroup, no patient with elevated sCD147 died or was diagnosed with disease recurrence in the follow-up period. All sCD147 > 24ng/mL patients in this subgroup were BCLC stage A or B (except one BCLC stage C) with good liver function and sCD147 levels similar to the

control cohort. The CLD cohort showed no survival difference based on sCD147 levels. Furthermore, there was no correlation of sCD147 with AFP levels with CLD and/or HCC. Importantly, there appears to be progression from normal sCD147 levels, to levels that are depressed with cirrhosis then elevated with the subgroup with a poor HCC prognosis. This is 
highly clinically relevant as many individuals in Western countries have cirrhosis then proceed to develop HCC ${ }^{3}$. The association between CD147 and response to treatment was difficult to assess; patient numbers were small in each treatment subgroup, several received multiple treatment modalities. However, there was no change in patient outcome with high sCD147 levels irrespective of treatment modality. Additionally, rapid disease progression post-liver $=$ transplantation in a patient with significantly elevated pre-transplant sCD147 level deserves further investigation.

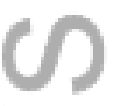

BCLC tumor staging ${ }^{23}$ has the best independent predictive power for survival rates compared to six other prognosis systems ${ }^{24}$. It links stage classification with a treatment strategy and defines standard of care for each stage, which transitions to another stage upon treatment failure or procedural contraindications rather than any predictive marker. Incorporating sCD147 levels > $24 \mathrm{ng} / \mathrm{mL}$ into the BCLC system predicted the median survival time of 1.5 months in the advanced stages of disease. This is considerably shorter than the reported median survival times of 11 and 3 months for BCLC stages C and D, respectively ${ }^{23}$. The significantly shorter survival time for BCLC stage C patients with sCD147 levels $>24 \mathrm{ng} / \mathrm{mL}$ was not seen for BCLC stage $\mathrm{D}$, due to our small patient numbers and an overall short survival time. Further, the utility of diagnostic markers in BCLC stage D is limited as supportive care is the best current management ${ }^{25}$. In BCLC stage C, the significant decrease in survival time is important as this group is still considered for ongoing, active treatment. More accurate survival information may allow futile therapies and associated side effects to be avoided.

Two previous studies investigating the utility of this biomarker have differing results, but the 
variety of cancers investigated, lack of sub-categorization by staging, as we have done, and measuring plasma or serum are confounding factors. The novel diagnostic utility we have described was not observed in the only other study measuring sCD147 in HCC patients ${ }^{11}$. The discrepancy is explained by a combination of fewer patients, no stratification based on treatment response, many other cancer types included in their analysis, and serum was analyzed while we used plasma. This is important, given the known binding of CD147 to fibrin, which is removed in serum preparations ${ }^{26}$. In the pancreatic cancer study, sCD147 levels were significantly increased in pancreatic adenocarcinoma patients compared to healthy controls and chronic pancreatitis patients ${ }^{10}$. These results are compatible with ours as pancreatic adenocarcinoma patients also have a short survival time from diagnosis due to its high likelihood for early spread.

The strengths of this study are its multi-center, prospective design with large patient numbers. The inclusion of a CLD and non-liver disease control cohort are important control comparators. Its limitations are that there are differences between the CLD and HCC cohorts on demographics known to be associated with HCC (viral etiology, age). However, this was not significant in our analysis. Research arising from the results of this study need to address what role sCD147 levels have in predicting response to therapies and whether sCD147 levels are predictive of liver transplant patients who will transition beyond transplant criteria. The findings will also need to be validated prospectively in other large HCC populations.

In conclusion, sCD147 is a new prognostic HCC assessment. sCD147 plasma levels $>24 \mathrm{ng} / \mathrm{mL}$ predict 90-day mortality in the HCC without CR subgroup with good sensitivity and specificity. Furthermore, elevated sCD147 levels have an excellent specificity for predicting HCC 
progression. Incorporating sCD147 levels $>24 \mathrm{ng} / \mathrm{mL}$ into the BCLC staging system will identify a subgroup of patients with short survival and a low likelihood of response to current treatment.

Acknowledgement: This study was funded by the Australian Government National Health and Medical Research Council Grant ID 512283.

Conflicts of interest: The authors do not have any conflicts of interest to disclose.

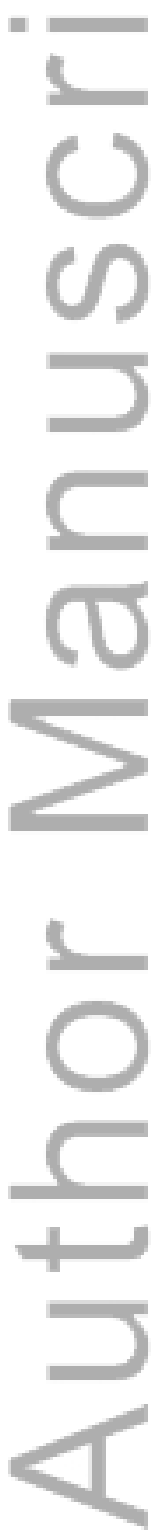




\section{REFERENCES}

[1] Jemal A, Siegel R, Ward E, Hao Y, Xu J, Thun MJ. Cancer statistics, 2009. CA Cancer J Clin. 2009; 59: 225-49.

[2] Dalhoff K, Dancey J, Astrup L, et al. A phase II study of the vitamin D analogue Seocalcitol in patients with inoperable hepatocellular carcinoma. Br J Cancer. 2003; 89: 252-7.

[3] Stroffolini T, Trevisani F, Pinzello G, et al. Changing aetiological factors of hepatocellular carcinoma and their potential impact on the effectiveness of surveillance. Digestive and liver disease : official journal of the Italian Society of Gastroenterology and the Italian Association for the Study of the Liver. 2011; 43: 875-80.

[4] Intasai N, Mai S, Kasinrerk W, Tayapiwatana C. Binding of multivalent CD147 phage induces apoptosis of U937 cells. Int Immunol. 2006; 18: 1159-69.

[5] Yoshida S, Shibata M, Yamamoto S, et al. Homo-oligomer formation by basigin, an immunoglobulin superfamily member, via its N-terminal immunoglobulin domain. Eur J Biochem. 2000; 267: 4372-80.

[6] Gou X, Ru Q, Zhang H, et al. HAb18G/CD147 inhibits starvation-induced autophagy in human hepatoma cell SMMC7721 with an involvement of Beclin 1 down-regulation. Cancer Sci. 2009; 100: 837-43.

[7] Qian AR, Zhang W, Cao JP, et al. Downregulation of CD147 expression alters cytoskeleton architecture and inhibits gelatinase production and SAPK pathway in human hepatocellular carcinoma cells. J Exp Clin Cancer Res. 2008; 27: 50.

[8] Tang Y, Nakada MT, Kesavan P, et al. Extracellular matrix metalloproteinase inducer stimulates tumor angiogenesis by elevating vascular endothelial cell growth factor and matrix metalloproteinases. Cancer Res. 2005; 65: 3193-9.

[9] Tsai WC, Chao YC, Lee WH, Chen A, Sheu LF, Jin JS. Increasing EMMPRIN and matriptase expression in hepatocellular carcinoma: tissue microarray analysis of immunohistochemical scores with clinicopathological parameters. Histopathology. 2006; 49: 38895.

[10] Zhang W, Erkan M, Abiatari I, et al. Expression of extracellular matrix metalloproteinase inducer (EMMPRIN/CD147) in pancreatic neoplasm and pancreatic stellate cells. Cancer Biol Ther. 2007; 6: 218-27.

[11] Moonsom S, Tayapiwatana C, Wongkham S, Kongtawelert P, Kasinrerk W. A Competitive ELISA for quantifying serum CD147: reduction of soluble CD147 levels in cancer patient sera. Hybridoma (Larchmt). 2010; 29: 45-52.

[12] Millimaggi D, Mari M, D'Ascenzo S, et al. Tumor vesicle-associated CD147 modulates the angiogenic capability of endothelial cells. Neoplasia. 2007; 9: 349-57.

[13] Sidhu SS, Mengistab AT, Tauscher AN, LaVail J, Basbaum C. The microvesicle as a vehicle for EMMPRIN in tumor-stromal interactions. Oncogene. 2004; 23: 956-63.

[14] Egawa N, Koshikawa N, Tomari T, Nabeshima K, Isobe T, Seiki M. Membrane type 1 matrix metalloproteinase (MT1-MMP/MMP-14) cleaves and releases a 22-kDa extracellular matrix metalloproteinase inducer (EMMPRIN) fragment from tumor cells. J Biol Chem. 2006; 281: 37576-85.

[15] Xu J, Shen ZY, Chen XG, et al. A randomized controlled trial of Licartin for preventing hepatoma recurrence after liver transplantation. Hepatology. 2007; 45: 269-76. 
[16] Zhang Q, Chen X, Zhou J, et al. CD147, MMP-2, MMP-9 and MVD-CD34 are significant predictors of recurrence after liver transplantation in hepatocellular carcinoma patients. Cancer Biol Ther. 2006; 5: 808-14.

[17] Chen Z, Liu, Y. Monoclonal antibody HAb18 to human hepatoma. Monoclonal Antibodies. 1990; 8.

[18] Lencioni R, Llovet JM. Modified RECIST (mRECIST) assessment for hepatocellular carcinoma. Seminars in liver disease. 2010; 30: 52-60.

[19] Therasse P, Arbuck SG, Eisenhauer EA, et al. New guidelines to evaluate the response to treatment in solid tumors. European Organization for Research and Treatment of Cancer, National Cancer Institute of the United States, National Cancer Institute of Canada. Journal of the National Cancer Institute. 2000; 92: 205-16.

[20] Forner A, Reig ME, de Lope CR, Bruix J. Current strategy for staging and treatment: the BCLC update and future prospects. Seminars in liver disease. 2010; 30: 61-74.

[21] Nikooie R, Rajabi H, Gharakhanlu R, et al. Exercise-induced changes of MCT1 in cardiac and skeletal muscles of diabetic rats induced by high-fat diet and STZ. J Physiol Biochem. 2013; 69: 865-77.

[22] Wanaguru M, Liu WM, Hahn BH, Rayner JC, Wright GJ. RH5-Basigin interaction plays a major role in the host tropism of Plasmodium falciparum. P Natl Acad Sci USA. 2013; 110: 2073540.

[23] Bruix J, Llovet JM. Prognostic prediction and treatment strategy in hepatocellular carcinoma. Hepatology. 2002; 35: 519-24.

[24] Marrero JA, Fontana RJ, Barrat A, et al. Prognosis of hepatocellular carcinoma: comparison of 7 staging systems in an American cohort. Hepatology. 2005; 41: 707-16. [25] Kejner AE, Burch MB, Sweeny L, Rosenthal EL. Bone Morphogenetic Protein 6 Expression in Oral Cavity Squamous Cell Cancer is Associated With Bone Invasion. Laryngoscope. 2013; 123: 3061-5.

[26] $\mathrm{Li} \mathrm{Y,Wu} \mathrm{J,} \mathrm{Song} \mathrm{F,} \mathrm{et} \mathrm{al.} \mathrm{Extracellular} \mathrm{membrane-proximal} \mathrm{domain} \mathrm{of} \mathrm{HAb18G/CD147}$ binds to metal ion-dependent adhesion site (MIDAS) motif of integrin beta1 to modulate malignant properties of hepatoma cells. J Biol Chem. 2012; 287: 4759-72.

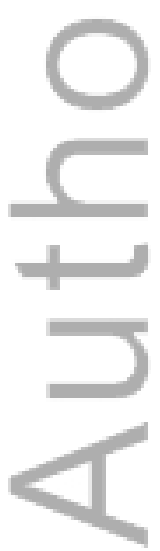




\section{FIGURE LEGENDS}

\section{Figure $1 \quad$ Study population stratification.}

Patient numbers per study group with Child-Turcotte-Pugh score and Barcelona Clinic Liver Cancer (BCLC) staging. There was a significant difference in Child-Turcotte-Pugh score C between the chronic liver disease (CLD) and hepatocellular carcinoma (HCC) cohorts $(P<0.05)$.

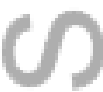

Figure 2 Correlation of sCD147 with mortality in CLD and HCC.

sCD147 and mortality is shown in; A) CLD and HCC and B) HCC patients with BCLC stage A and B compared to stage C and D. Mortality correlated with sCD147 levels in HCC patients $(P=0.031)$ and patients with BCLC Stage C and D disease $(P=0.045)$.

Figure 3 sCD147 distinguishes outcome in HCC and BCLC Stage C disease. Patient survival according to sCD147 levels in the HCC cohort and HCC patients with BCLC stage C disease. There was a significant decrease in the survival time of patients with sCD147 $>24 \mathrm{ng} / \mathrm{mL}$ in the HCC cohort $(P=0.041)$ and the patients with BCLC stage C disease $(P=0.030)$. BCLC stage $\mathrm{D}$ is not shown as there were only 3 individuals in this group with sCD147 > 24ng/mL.

Figure 4 Survival curve for HCC cohort stratified by sCD147 levels. Kaplan-Meier survival curves for the HCC cohort, stratified for sCD147 levels > $24 \mathrm{ng} / \mathrm{mL}$ or $\leq 24 \mathrm{ng} / \mathrm{mL}$. The overall difference was not significant $(P=0.66)$ but the 
difference in 90-day survival and 180-day survival was significant $(P<0.01$ and $P<0.05$, respectively).

Figure 5 Immunohistochemistry of CD147 in non-diseased, cirrhotic and HCC liver sections.

Immunohistochemistry of CD147 expression in liver tissue. A and B) Non-diseased liver, $C$ and D) cirrhotic alcoholic liver disease (ALD), E and F) cirrhotic ALD with hepatocellular carcinoma (HCC), G) isotope control. CD147 is abundantly expressed on hepatocytes, leukocytes and biliary epithelium (arrow). There is negligible expression in the fibrous septa where activated hepatic stellate cells are located ( $\dagger$ ).

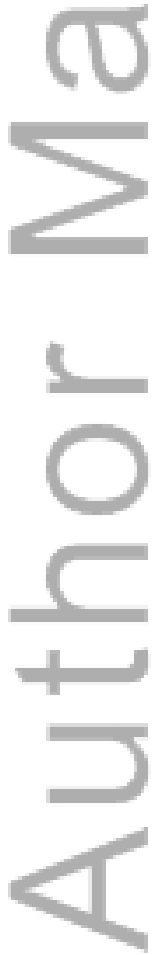


TABLES

Table $1 \quad$ Patient demographics and liver disease characteristics.

\begin{tabular}{|c|c|c|c|c|c|c|c|}
\hline \multirow{2}{*}{ Variable } & \multicolumn{2}{|c|}{ Control } & \multicolumn{2}{|c|}{ CLD } & \multicolumn{2}{|c|}{ HCC } & \multirow{2}{*}{ P-value } \\
\hline & $\mathrm{n}$ & $\%$ & $\mathrm{n}$ & $\%$ & $\mathrm{n}$ & $\%$ & \\
\hline Total & 52 & 18.8 & 115 & 41.5 & 110 & 39.7 & \\
\hline Male & 41 & 78.9 & 90 & 78.3 & 95 & 86.4 & \\
\hline Age, years & \multicolumn{2}{|c|}{$67.1(11.3)$} & \multicolumn{2}{|c|}{$59.3(11.5) \dagger$} & \multicolumn{2}{|c|}{$63.0(10.4)$} & $<0.001$ \\
\hline median (IQR) & \multicolumn{2}{|c|}{$28.0(24.5-32.6)$} & \multicolumn{2}{|c|}{$27.8(24.9-30.9)$} & \multicolumn{2}{|c|}{$25.9(23.6-29.5) \dagger \ddagger$} & 0.008 \\
\hline Waist circumference, $\mathrm{cm} \quad$ mean (SD) & \multicolumn{2}{|c|}{$93.9(12.5)$} & \multicolumn{2}{|c|}{$96.3(13.9)$} & \multicolumn{2}{|c|}{$96.2(13.6)$} & 0.50 \\
\hline $\begin{array}{rr}\text { Ethnicity }= & \begin{array}{r}\text { Anglo Saxon } \\
\text { Asian } \\
\text { Mediterranean } \\
\text { Other }\end{array} \\
\end{array}$ & $\begin{array}{c}27 \\
7 \\
12 \\
6 \\
\end{array}$ & $\begin{array}{l}51.9 \\
13.5 \\
23.1 \\
11.5\end{array}$ & $\begin{array}{l}61 \\
14 \\
23 \\
17\end{array}$ & $\begin{array}{l}53.0 \\
12.2 \\
20.0 \\
14.8\end{array}$ & $\begin{array}{c}54 \\
25 \\
25 \\
4\end{array}$ & $\begin{array}{c}49.1 \\
22.7 \\
22.7 \\
3.6\end{array}$ & \\
\hline History of diabetes & 16 & 30.8 & 39 & 33.9 & 37 & 33.6 & \\
\hline $\begin{array}{lr}\text { Underlying liver disease } & \text { Alcohol } \\
\text { HCV } \\
\text { HBV } \\
\text { NAFLD } \\
\text { Other } \\
\end{array}$ & & & $\begin{array}{l}35 \\
40 \\
12 \\
13 \\
15\end{array}$ & $\begin{array}{l}30.4 \\
34.8 \\
10.4 \\
11.3 \\
13.0 \\
\end{array}$ & $\begin{array}{c}19 \\
58 \\
18 \\
3 \\
12 \\
\end{array}$ & $\begin{array}{c}17.3 \\
52.7 \\
16.4 \\
2.7 \\
10.9 \\
\end{array}$ & \\
\hline CTP Score & & & \multicolumn{2}{|c|}{$7(5-10)$} & \multicolumn{2}{|c|}{$6(5-8)$} & 0.048 \\
\hline median (IQR) & & & \multicolumn{2}{|c|}{$9.4(3.6-16.9)$} & \multicolumn{2}{|c|}{$8.7(5.4-12.2)$} & 0.35 \\
\hline MELD $\geq 15$ & & & 35 & 30.4 & 11 & 10.0 & 0.41 \\
\hline$\frac{0}{\frac{\mathrm{A}}{\mathrm{B}}}$ & & & & & $\begin{array}{l}\frac{3}{34} \\
\frac{28}{32} \\
\underline{13}\end{array}$ & $\begin{array}{l}\frac{2.7}{30.9} \\
\frac{25.5}{29.1} \\
\underline{\underline{11.8}}\end{array}$ & \\
\hline 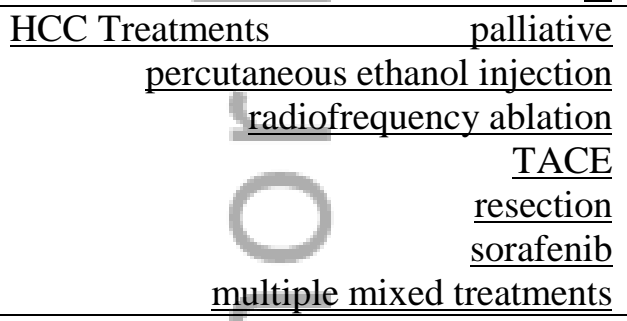 & & & & & $\begin{array}{l}\frac{3}{2} \\
\frac{14}{26} \\
\frac{26}{6} \\
\underline{6} \\
\underline{52} \\
\end{array}$ & $\frac{\frac{2.7}{1.8}}{\frac{12.7}{23.6}} \frac{\underline{6.4}}{\underline{5.5}}$ & \\
\hline
\end{tabular}

$\mathrm{CLD}=$ chronic liver disease, $\mathrm{HCC}=$ hepatocellular carcinoma, $\mathrm{BMI}=$ body mass index, $\mathrm{HCV}=$ hepatitis $\mathrm{C}$ virus, $\mathrm{HBV}=$ hepatitis $\mathrm{B}$ virus, NAFLD=non-alcoholic fatty liver disease, $\mathrm{CTP}=$ Child-Turcotte-Pugh, $\mathrm{MELD}=$ model for end stage liver disease, $\underline{B C L C=B a r c e l o n a}$ Clinic Liver Cancer, $\mathrm{TACE}=$ transcatheter arterial chemoembolization.

Values significantly different: $†$ compared to Control, $\ddagger$ compared to CLD.

This article is protected by copyright. All rights reserved. 


\begin{tabular}{|c|c|c|c|}
\hline Group & $\begin{array}{l}\text { Total cohort } \\
\text { sCD147 levels }\end{array}$ & $\underline{\mathrm{AFP}}>\mathbf{6 \mathrm { kIU } / \mathrm { L }}$ & $\underline{\mathrm{AFP}}>\mathbf{2 0 0} \mathrm{kIU} / \mathrm{L}$ \\
\hline CLD & $\frac{r=-0.04969}{\frac{P=0.5995}{(n=115)}}$ & $\begin{array}{l}4.35 \% \\
(n=5)\end{array}$ & $\underline{0 \%}$ \\
\hline$\underline{\mathrm{HCC}}$ & $\begin{array}{l}\mathrm{r}=0.1781 \\
\mathrm{P}=0.0665 \\
(\mathrm{n}=107)^{*}\end{array}$ & $\frac{53.64 \%}{(n=59)}$ & $\frac{21.82 \%}{(n=24)}$ \\
\hline
\end{tabular}

* Three patients with AFP > 50,000 where excluded as they were more than 3 standard deviations from the median.

$\underline{\mathrm{CLD}=\text { chronic liver disease, } \mathrm{HCC}=\text { hepatocellular carcinoma. }}$ 


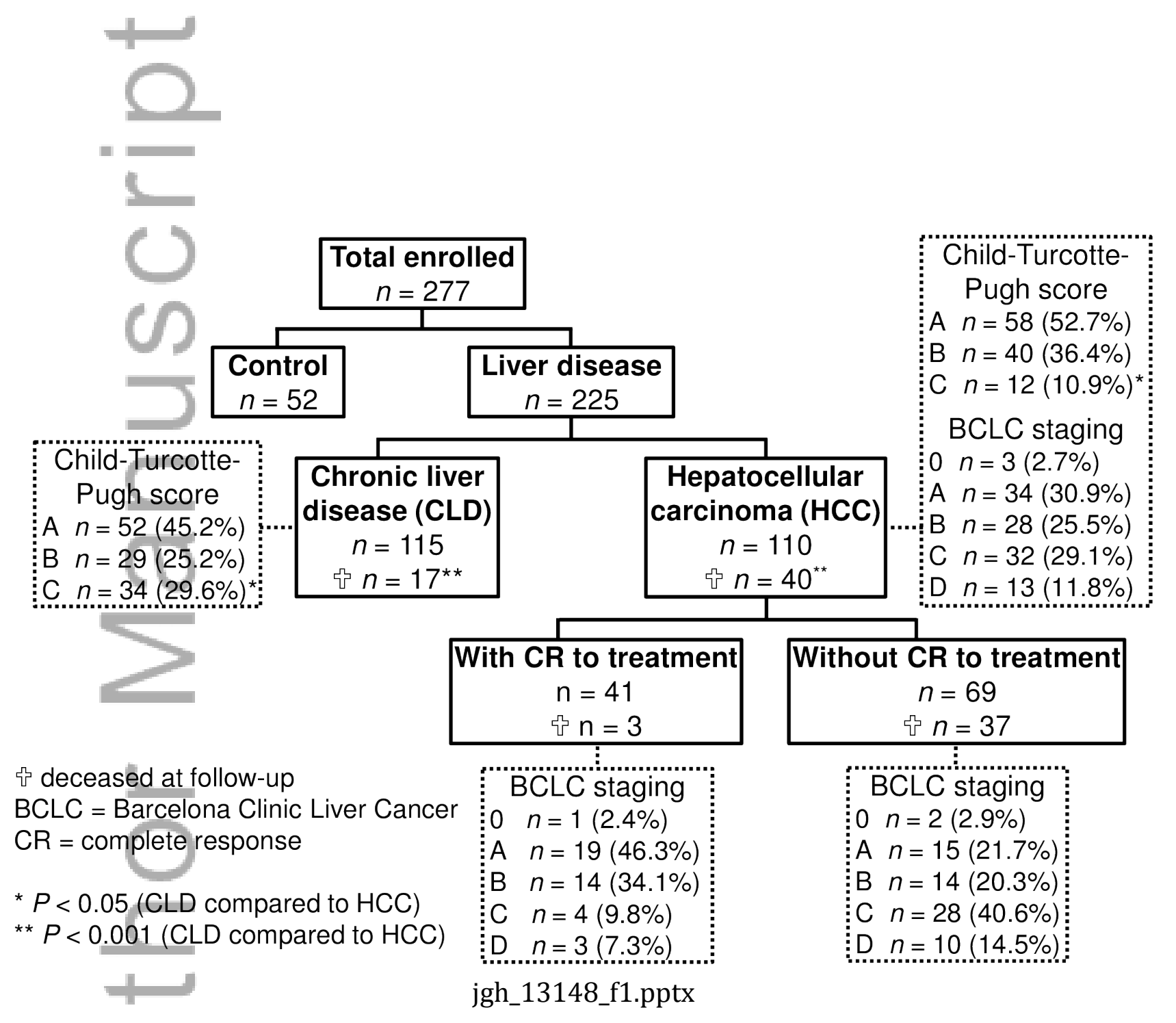

This article is protected by copyright. All rights reserved. 

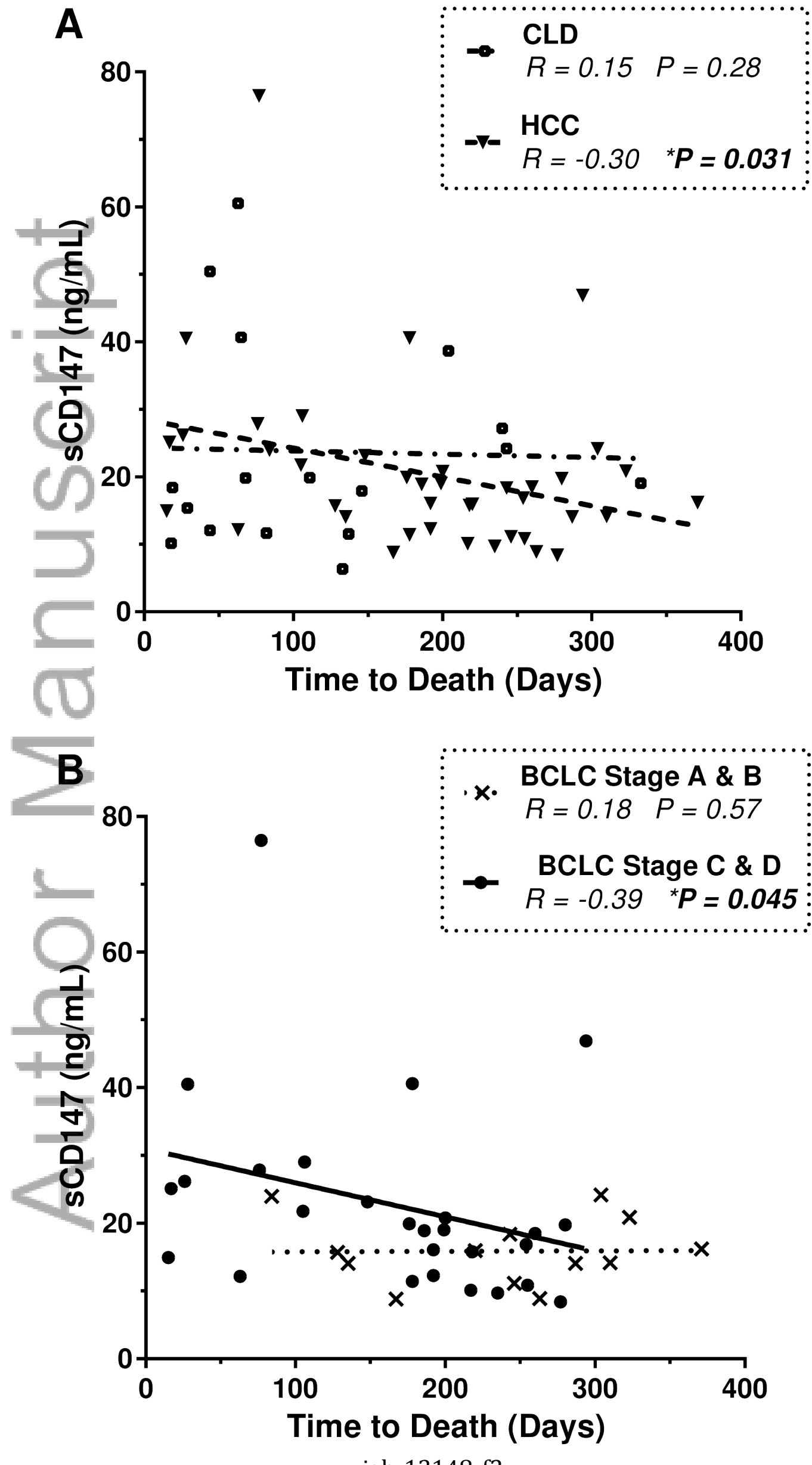

jgh_13148_f2 


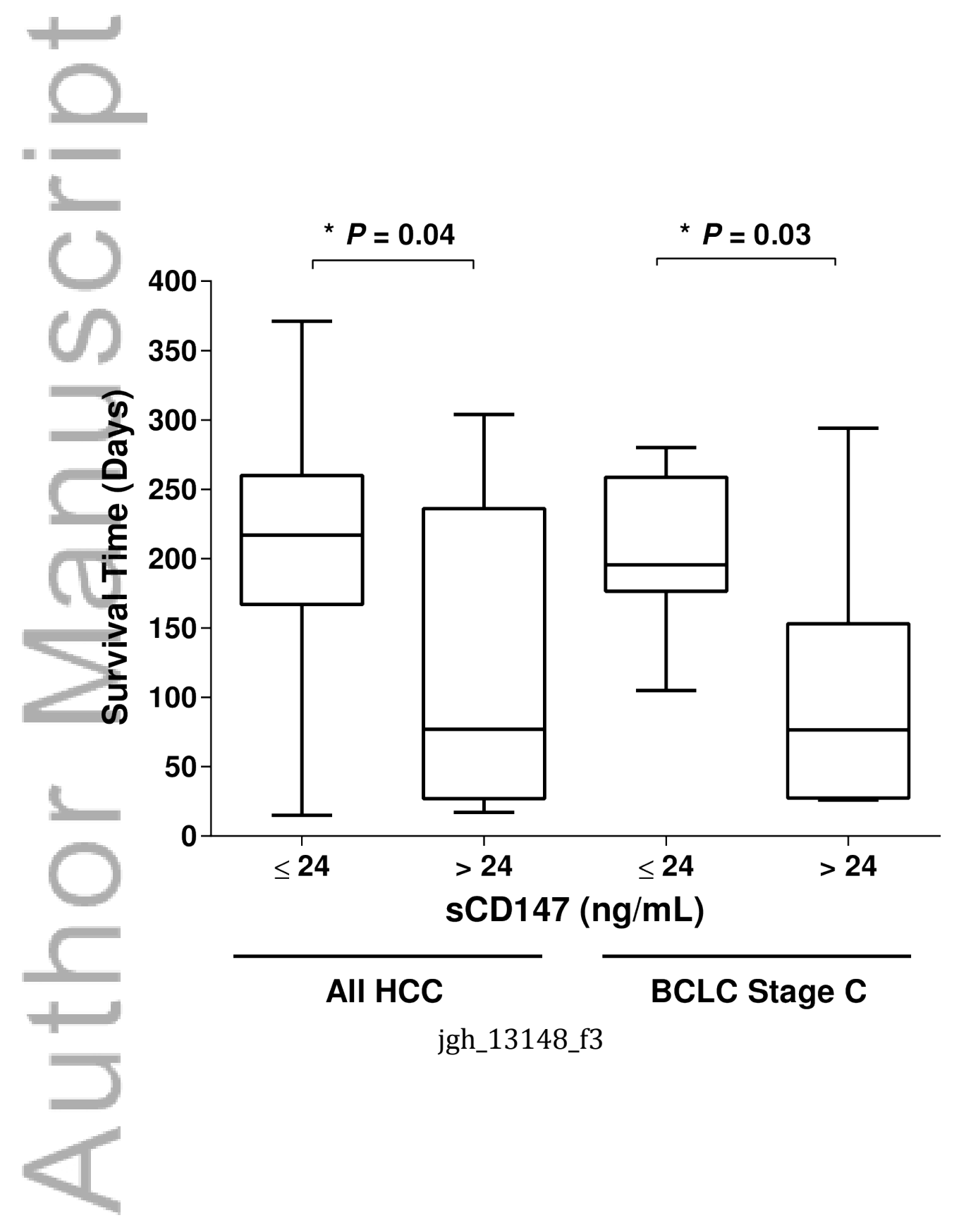

This article is protected by copyright. All rights reserved. 


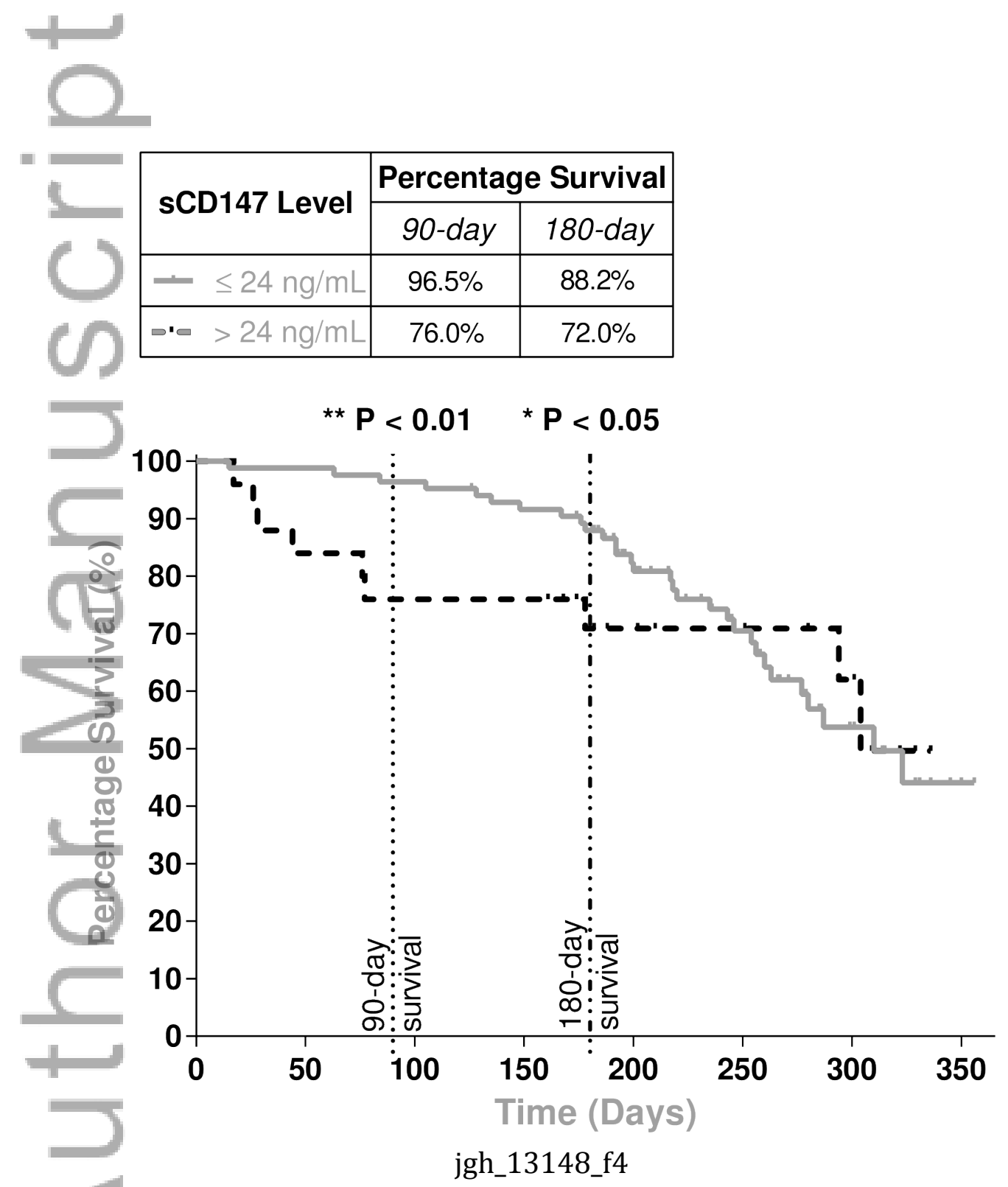

This article is protected by copyright. All rights reserved. 

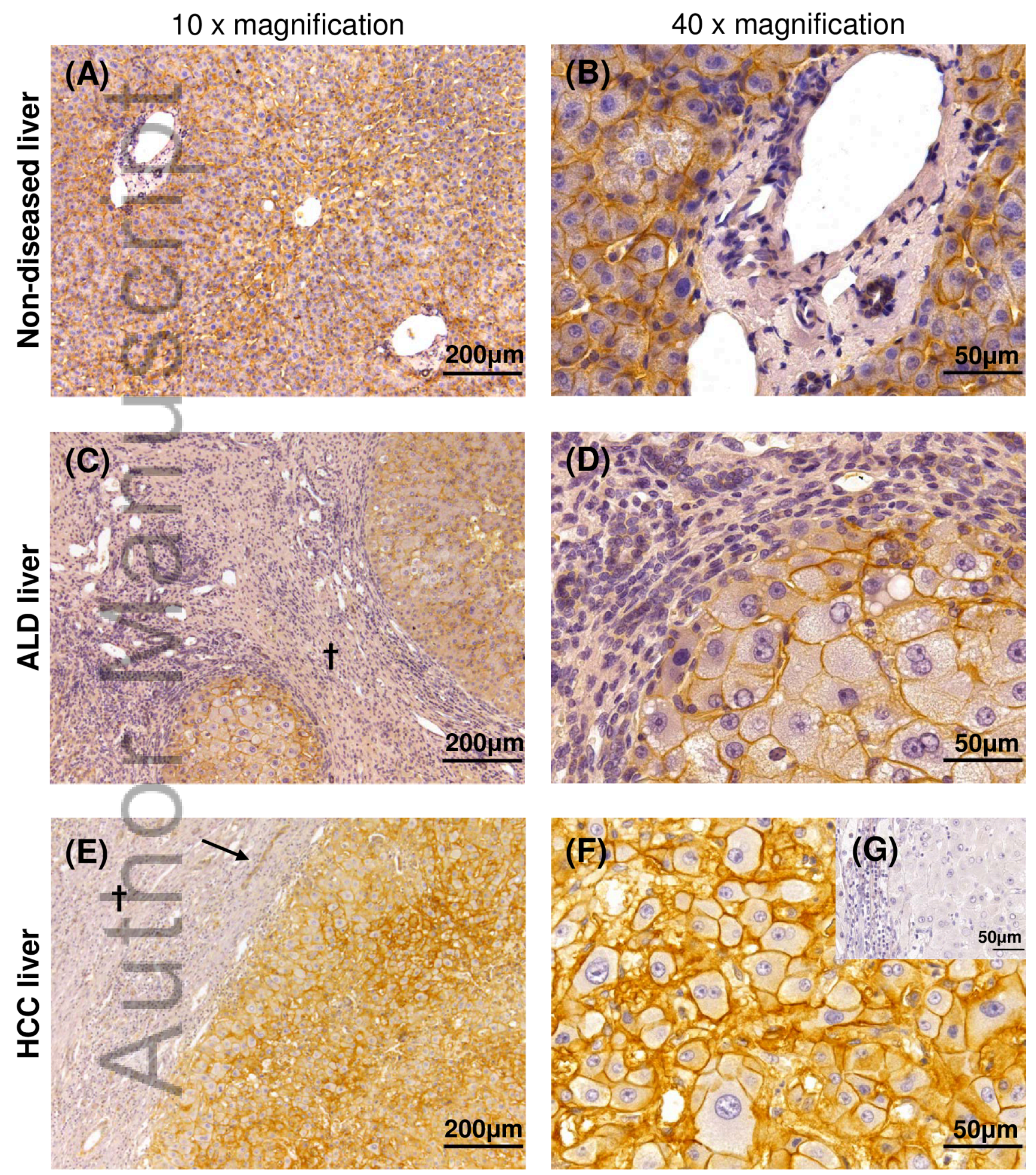

jgh_13148_f5.pptx 


\section{University Library}

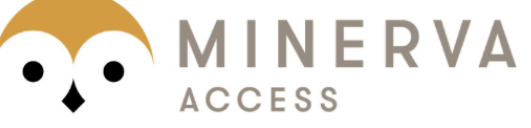

A gateway to Melbourne's research publications

Minerva Access is the Institutional Repository of The University of Melbourne

\section{Author/s:}

Lee, A;Rode, A;Nicoll, A;Maczurek, AE;Lim, L;Lim, S;Angus, P;Kronborg, I;Arachchi, N;Gorelik, A;Liew, D;Warner, FJ;McCaughan, GW;McLennan, SV;Shackel, NA

Title:

Circulating CD147 predicts mortality in advanced hepatocellular carcinoma

\section{Date:}

2016-02-01

\section{Citation:}

Lee, A., Rode, A., Nicoll, A., Maczurek, A. E., Lim, L., Lim, S., Angus, P., Kronborg, I., Arachchi, N., Gorelik, A., Liew, D., Warner, F. J., McCaughan, G. W., McLennan, S. V. \& Shackel, N. A. (2016). Circulating CD147 predicts mortality in advanced hepatocellular carcinoma. JOURNAL OF GASTROENTEROLOGY AND HEPATOLOGY, 31 (2), pp.459-466. https://doi.org/10.1111/jgh.13148.

Persistent Link:

http://hdl.handle.net/11343/290870 